\title{
Effect of Galactomannans and Low Esterified Pectin Combinations on Fruit Preparation Synersis, Rheology and Stability on Storage
}

\author{
Omprakash H. Nautiyal \\ India malt Pvt. Ltd., Vill. Savli, Taluk Manjusar, Vadodara, Gujarat, 371995, India
}

\begin{abstract}
The objective of the study was to evaluate the fruit preparation syneresis, rheology and stability of the various blends of galactomannans. Percent composition of low estreified pectin $(0.3 \%)$, semi refined cassia gum $(0.50 \%)$ guar gum $(0.50 \%)$, locust bean gum $(0.50 \%)$ by w/v were changed keeping the sugar consistent. Fruit preparations were heated at $40^{\circ} \mathrm{C}$ for mixing of dry blends. Finally the blend was heated at $85^{\circ} \mathrm{C}$ and the end temperature was hold for 5 minutes. It was then cooled down to below $70^{\circ} \mathrm{C}$, and evaporated water was added. It was cooled to $20^{\circ} \mathrm{C}$ and the viscosity was measured next day and after one week at 20 and $100 \mathrm{rpm}$. The blends were stored in freeze and viscosity was measured at $20^{\circ} \mathrm{C}$. The synersis was found to be satisfactory without any cream formation at the top of the blends. The enhancement in the values of the viscosity may be attributed to the arrangement of galactose: mannose series arrangement in the respective galactomannans and plays important role in the stabilization of the food formulations.
\end{abstract}

Keywords Low Esterified Pectin, Cassia Gum SR, Guar Gum, Locust Bean Gum, Synersis And Stability

\section{Introduction}

Hydrocolloids are either linear or branched. Most are linear, meaning they are composed of one long chain with small side chains. Branched hydrocolloids, composed of many branches joined together in a bushy shape, include tree gums such as gum Arabic and the starch amyl pectin. Counter intuitively, branched hydrocolloids typically display lower viscosities (lower viscosity means thinner; higher viscosity means thicker) than linear hydrocolloids of the same size. Branched hydrocolloids are compact, acting like tennis balls, while linear hydrocolloids act like a tangle of spaghetti moving about in solution, taking up more space. For a given hydrocolloid, larger molecules having more sugar units will produce higher viscosities.

The main reason for adding a gum or hydrocolloid to a food product is to improve its overall quality. This improvement may relate to its appearance, convenience, stability, cost, texture, etc., all of which are eventually judged by the consumer. The consumer usually judges the product by simply eating it and seeing how it tastes. At this point he does not care if the added gum is a galactomannan or a sulphated galactan, nor does he worry about whether the vitamin $\mathrm{A}$ in the product is naturally derived from sharks liver or

* Corresponding author:

opnautiyalus@yahoo.com (Omprakash H. Nautiyal)

Published online at http://journal.sapub.org/food

Copyright (C) 2012 Scientific \& Academic Publishing. All Rights Reserved synthesized from $\mathrm{b}$ ionone. He is concerned with the taste and mouth feel of the product. If it tastes good, he is happy if it tastes bad, he is unhappy, and so eventually is the manufacturer of that specific food item.

Viscosity is the resistance to flow of a liquid system. In colloidal suspensions it is increased by the thickening of the liquid phase as a result of liquid absorption and consequent swelling of the dispersed colloid. This thickening, or viscosity producing, effect of gums in food products is, in turn, responsible for other functional effects such as the suspension of solid particles, the emulsification of oil and water phases, the stabilization of liquid solid gas phases, the dispersion of solid and liquid phases, and related phenomena.

When hydrocolloids are used as viscosity producing agents for the purpose of suspending, emulsifying, or stabilizing a food system, shelf stability is extremely important and the selection of the proper hydrocolloid is critical. Degradation of the hydrocolloid and the resulting reduction in the viscosity of polymer solutions may impair the flow properties and appearance of the product sufficiently to reduce its consumer acceptability.

${ }^{1}$ The quality criteria of fruit preparations are mainly determined by their rheological flow behaviour. The formulation of the fruit preparation as well as the thickening agents

\footnotetext{
${ }^{1}$ The research was conducted from business point of view so that the in house Cassia gum along with outsourced hydrocolloids may find greater commercial application. It is well known that hydrocolloids are in great demand worldwide in the application of stabilizing the food from synersis and rheology point of view. The study was found to be useful employing the fruit preparation from Austria
} 
used has a decisive effect on the flow behaviour. The mixing behaviour with the milk product and the syneresis behaviour also affect the quality of yoghurt fruit preparations. Pectins for fruit preparations, such as Low Methyl ester Classic Apple Pectins and Low Methyl ester Amidated Pectins from H\&F (Figure 1), are systematically standardized to specific flow behaviour. Thus if the appropriate pectin is selected for a pre-determined formulation, optimum products are obtained. ${ }^{1,2-3}$

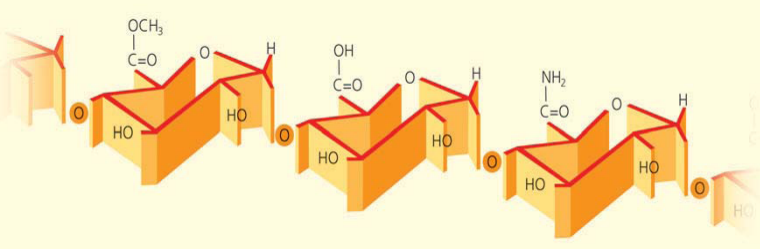

Figure 1. Molecular structure of esterified pectin

The flow behaviour of yoghurt fruit preparations can be characterized by determining the following parameters, using, for example, a shear-stress controlled rheometer: Viscosity Yield point and Regenerative capacity.

Viscosity is the property of substances indicating their resistance to flow. Fruit preparations are shear-thinning, pseudo plastic substances where viscosity drops with increasing shear stress (shear rate). Therefore, shear-thinning products are subject to a drop in viscosity (shear-thinning) during processing due to stress (pumping, stirring). During the idle state or when exposed too little stress, these products have a high viscosity. As yoghurt fruit preparations have to be frequently pumped or stirred during the manufacturing process, the level of the stress-dependent viscosity is an important factor. The shear stress (shear rate) that can act on the fruit preparation differs depending on the production facility. In the case of pumping it depends on the pipe geometry and the pump's delivery rate, with stirring it is mainly determined by the stirring speed and the container geometry ${ }^{1,2-3}$

If the viscosity is low during pumping and stirring, not only is less force and energy required executing the process but the product is also prevented from damage due to the smaller force exerted, so that fruits or fruit components are better preserved.

Gums as food constituents or as food additives can influence processing conditions in the following ways: retention of water reduction of evaporation rates alteration of freezing rates modification of ice crystal formation participation in chemical reactions. These functional effects are not isolated phenomena, which can be followed easily they are only evident in the textural qualities or rheological behaviour of the final product. While the functional effects of a gum are its most important characteristics in determining its use in foods, these effects must be considered in context with many other factors, including price, availability, ease of handling, and legal restrictions related to their use.

Gums are used in a wide range of specific food applications, ranging from adhesives to whipping agents. Typical specific functions and food applications are shown in Table 1, but the general function of gums can be limited to their two major properties gelling and thickening.

All gums, or hydrocolloids, by definition and usage have a thickening, or viscosity producing, effect when dispersed in a water medium. This property is the basis for their use as bodying, stabilizing, and emulsifying agents in many foods. A comparatively few of the important gums i.e., agar, algin, carrageenan, furcellaran, gelatin, pectin, and starch also have the ability to form gels under specific conditions of use. Gels, when referring to foods, are products that will retain their shape and will not flow unless pressure is applied. Probably the most common gelled food product is gelatin dessert gel, which has enjoyed enormous popularity in this country for many years. Other well known food gels are starch based milk puddings, gelatin aspics, and pectin gelled cranberry sauce. In Europe, milk puddings of the Blanc mange type are very popular and are usually made with seaweed extracts of the carrageenan or alginate type.

There is interplay between the viscosity and gelling characteristics of any specific gum and these factors must be taken into consideration when these gums are used for example, depending on the type of gelatin and on its concentration, gelatin can be used as a thickening agent rather than a gelling medium. In a related way, temperature also plays an effect as the temperature increases, the viscosity decreases, thus decreasing the effective thickening properties of the gum. This temperature increase is in most cases the same as an effective reduction in gum concentration. ${ }^{1,2-3}$

To determine the structural disintegration of a fruit preparation during processing, the fruit preparation is sheared under defined conditions after determining the yield point before shearing. By shearing, the gelled portion is irreversibly destroyed. Immediately afterwards, the so-called "yield point after shearing" is determined within the sample. The yield point after shearing is a measure for the texture of fruit preparations immediately after a step in production. The difference between yield point before shearing and yield point after shearing describes the overall structural disintegration of a fruit preparation on account of the shear stress applied. If, after a given resting phase, another yield point measurement is conducted, the so-called "regenerated yield point" is obtained. The regenerated yield point is a measure for the texture of fruit preparations after these have been subjected to a defined mechanical stress and, subsequently, after a defined regenerative phase. The regenerated yield point specifies the texture of fruit preparations. ${ }^{4,5}$

\section{Materials and Method}

Low esterified pectin, Locust bean gum and Fruit concentrate were purchased from the international suppliers. Guar gum was purchased from Shriram Hydrocolloids Corporation. Semi refined cassia gum was manufactured in house. Sugar was purchased from India.

Dry ingredients were mixed and pre blend was prepared. 
The fruit preparation was heated to $40^{\circ} \mathrm{C}$. Pre blend was dispersed into the fruit preparation. It was then heated up to minimum $85^{\circ} \mathrm{C}$. When the time was found too long the heating was stopped at $85^{\circ} \mathrm{C}$ and the end temperature was noted. The lumps formation was observed during dispersion of pre blend. The heating was further hold for 5 minutes and then allowed to cool down below $70^{\circ} \mathrm{C}$. The evaporated water was compensated. It was finally cooled down to $20^{\circ} \mathrm{C}$.

The viscosity was measured next day and after one week at 20 and $100 \mathrm{rpm}$. The blends were stored in the freeze and were measured at $20^{\circ} \mathrm{C}$. The stability test of the prepared blend was tested after one week. The blends were stored in the freeze at $<80^{\circ} \mathrm{C}$. The temperature was monitored regularly and assured to be at aforesaid temperature ${ }^{6,7}$

Since most gums are long chain polymers, they are subject to the type of molecular breakdown caused by cleavage of molecular bonds, resulting in lower viscosities. Determination of the exact causes of degradation or loss of viscosity is often difficult. Frequently, polymers are degraded by the use of high shearing equipment used to put them into solution, or by the high temperatures used in processing. In general, the low viscosity natural gums are more stable than the high viscosity types. Studies on the comparative stabilities of gums are more valid if comparisons are made between solutions of equal viscosity rather than of equal concentrations, which is so often the case.

\subsection{Stability Tests after Week}

The blends were evaluated for synersis, sedimentation at the bottom, layer on the top of the blends; creaminess of the blends (may contain destabilized particles). Most important was homogeneous phase observation. Seldom do processors immediately think of gums when working with fluid products. The truth is, however, that they can be helpful in these products at low concentrations. Most obviously, the viscosity of flavoured milk can be increased and maintained through the addition of a gum. Less obvious, is the stabilizer's ability to maintain particulates in suspension.

Flavoured milk products contain sweeteners and flavouring agents such as cocoa powder. More often than not, these ingredients are not dissolved, but merely suspended. Eventually, gravity causes them to settle out.
At low concentrations, a stabilizer can help keep the ingredients suspended without making the product too thick. This works in two ways:

First, a stabilizer, such as starch, builds viscosity making it take longer for gravity to pull the particles out of the suspension. Second, if a gum like carrageenan is used, a colloid network exists even though the concentration is low enough to prevent actual gelling. This network supports particulate matter and prevents settling.

Working on the frost line

Distribution and handling exposes hard frozen products to a wide variety of environmental and temperature changes. This exposure to temperature fluctuation is known as "heat shock". $8-9$

\section{Results and Discussion}

The study was firstly carried out blending $97 \%$ of fruit concentrate and $3 \%$ of sugar and on testing its viscosity at 20 rpm and $100 \mathrm{rpm}$, (Table 1 and 2) it was found to be 600 and $372 \mathrm{mPas}$. The preparation did not form gel. It was thin and no synersis was found occur. Neither layer was found on top of it or the sedimentation at the bottom. It was observed to be homogeneous and no cream formation occurred. $0.50 \%$ of cassia gum SR when mixed with $96.50 \%$ of fruit concentrate viscosity of the blend was comparatively high at $20 \mathrm{rpm}$ than that at $100 \mathrm{rpm}$. But when $0.30 \%$ of low esterified pectin along with $0.50 \%$ cassia gum SR was mixed with $96.20 \%$ of fruit concentrates the viscosity of the blend was appreciably high at both the rpm. Locust bean gum as alone and while mixed with $0.3 \%$ of LEP with 96.50 and $96.20 \%$ fruit preparation found to exhibit the highest viscosities during entire study of the blend preparation. Though the use of LBG alone and/or with LEP found to yielding thicker blend without any synersis, and remain homogeneous but with the cream formation on the top of the blends. As observed from the table preparations from 2-6 found to yielding the blends with medium phase behaviour and without synersis, sedimentation, layer on the top. They were remained homogeneous even after a week and no cream formation was resulted.

Table 1. Rheological evaluation of Fruit Preparation with galactomannans

\begin{tabular}{|c|c|c|c|c|c|c|c|c|c|}
\hline SNo. & $\begin{array}{c}{ }^{1} \text { Pectin } \\
\%\end{array}$ & $\begin{array}{c}{ }^{2} \text { Cassia } \\
\text { gum SR\% }\end{array}$ & $\begin{array}{c}{ }^{3} \text { Guar } \\
\text { gum\% 200 }\end{array}$ & $\begin{array}{c}{ }^{4} \text { LBG } \\
\text { L147\% }\end{array}$ & Sugar\% & ${ }^{5}$ Fruit prep. \% & Total\% & $\begin{array}{c}\text { Viscosity meas- } \\
\text { urement mPas }\end{array}$ & \\
\hline & & & & & & & & Spindle L4 20 rpm & Spindle L4 100 rpm \\
\hline 1 & - & - & - & - & 3.00 & 97.00 & 100 & 600 & 372 \\
\hline 2 & 0.30 & - & - & - & 3.00 & 96.70 & 100 & 1860 & \\
\hline 3 & - & 0.50 & - & - & 3.00 & 96.50 & 100 & 3089 & 960 \\
\hline 4 & 0.30 & 0.50 & - & - & 3.00 & 96.20 & 100 & 3629 & 1260 \\
\hline 5 & - & - & 0.50 & - & 3.00 & 96.50 & 100 & 1290 & \\
\hline 6 & 0.30 & - & 0.50 & - & 3.00 & 96.20 & 100 & 2010 & 588 \\
\hline 7 & - & - & - & 0.50 & 3.00 & 96.50 & 100 & 8096 & 894 \\
\hline 8 & 0.30 & - & - & 0.50 & 3.00 & 96.20 & 100 & 9868 & 2849 \\
\hline
\end{tabular}

${ }^{1}$ Pectin low esterified (Cargill)

${ }^{2}$ Cassia gum semi refined (in house)

${ }^{3}$ Guar gum (Unipectin)

${ }^{4}$ Locust bean gum (Danisco)

${ }^{5}$ Fruit preparation (Austria) 
Table 2. Fruit preparations sensorial evaluation

\begin{tabular}{|c|c|c|c|c|c|c|}
\hline Gel formation & Liquid/thin/medium/thick & ${ }^{1}$ Synersis & Layer on top & Sedimentation at bottom & homogeneous & ${ }^{2} \mathrm{FP}$ creamy \\
\hline No & Thin & No & No & No & Yes & No \\
\hline No & Medium & No & No & No & Yes & No \\
\hline No & Medium & No & No & No & Yes & No \\
\hline No & Medium & No & No & No & Yes & No \\
\hline No & Medium & No & No & No & Yes & No \\
\hline No & Medium & No & No & No & Yes & No \\
\hline No & Thick & No & Yes & No & Yes & No \\
\hline No & Thick & No & Yes & No & Yes & No \\
\hline
\end{tabular}

${ }^{1}$ it is the phase separation when no balanced synergy occurs. ${ }^{2}$ Fruit preparation

Table 3. Viscosity measure Data Analysis

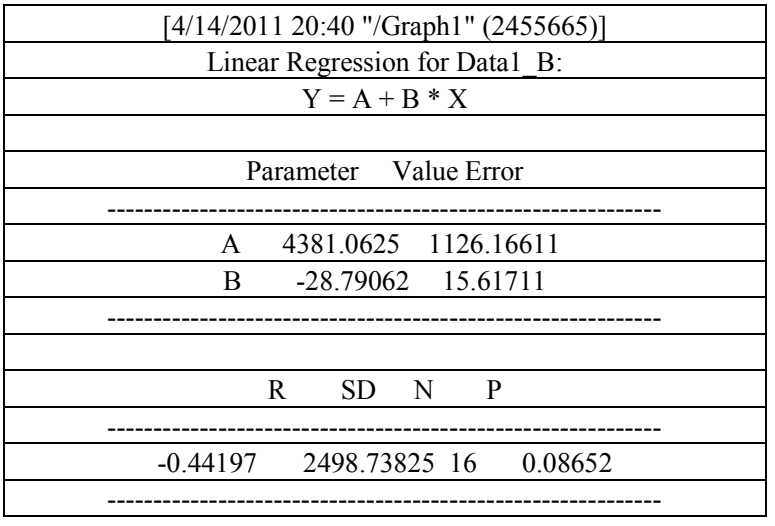

Table 3 specifically shows the statics of the experimental graph plotted and it may be observed that the viscosity at 100 rpm was lesser to that of the values of viscosity at $20 \mathrm{rpm}$ indicating the major role played by the shear effect on the fruit preparation that were stabilized employing galactomannans. ${ }^{6,7-8}$

This behaviour of the blends studied using these galactomannans clearly indicated that their functional structure plays an important role in imparting the rheology of the studied fruit preparations. Also low esterified pectin contains a modified functional structure and profoundly contributed in the stabilities of the studied blends either alone or in combinations of other studied galactomannans. The incubation of the fruit blends at $20^{\circ} \mathrm{C}$ over a week exhibited satisfactory results owed to their behaviour. ${ }^{9-10}$

Syneresis is the release of liquid from gels, especially after mechanical destruction of the gel network. In fruit preparations syneresis is undesirable. Especially in layered products, where the release of liquid is likely to discolour the white milk product, it is important to use fruit preparations showing little or no tendency to syneresis. To determine syneresis behaviour, a defined amount of fruit preparation is mechanically destroyed under specified conditions and then filled into a funnel. The released liquid is collected in a test tube or measuring glass. The lower the amount of liquid released from the test fruit preparation over a specified time period, the lower its tendency to syneresis (Figure 2, 3).

Pectin is an important structure-giving element of all plant foods. Chemically speaking, pectin is a macromolecular compound belonging to the hetero polysaccharides. Its main component is polygalacturonic acid, which is partly esterified with methanol. Pectins with a degree of esterification of $50 \%$ or more are known as high methyl ester pectins, whereas pectins of a $\mathrm{DE}$ below $50 \%$ are known as low methyl ester pectins

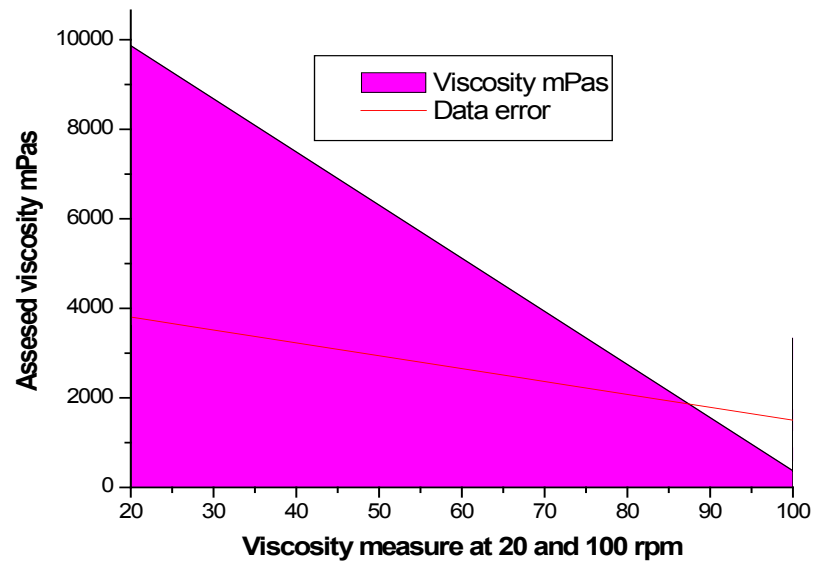

Figure 2. Rheological behaviour of fruit preparation with galactomannans

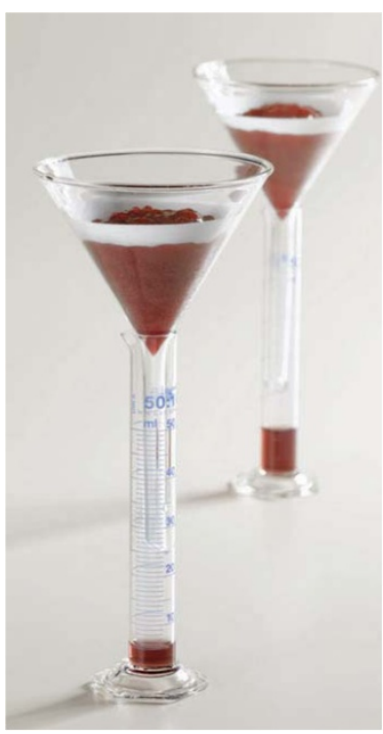

Figure 3. Synersis test for fruit preparations

The setting temperature of a gel preparation, i.e. the temperature at which the gelation of pectin gels starts during the cooling phase after the heating process has been completed, is an important parameter. At this point, a "sol-gel" transition takes place, i.e. the pectin chains arrange them- 
selves in a three-dimensional network. The setting temperature determines the filling temperature, depending on whether pre-gelation is to be prevented or is specifically intended. Pre-gelation always occurs if the filling temperature is below the setting temperature. In this case, a gel network forms even before depositing and is then again destroyed by mechanical stress during the filling process. This results in a partial loss of the overall reachable gel strength and in an increased tendency to syneresis.

Most hydrocolloids have important side groups on their main sugar backbone chain. These side groups affect solubility, gelling ability, susceptibility to salts, etc. Agar and different types of carrageenan (which have very different properties) differ only in their side chain groups. All cellulose derivatives have the same backbone: a cellulose molecule, with different side chains. Sometimes two different hydrocolloids differ only in the distribution of their side chains; the very different properties of locust bean gum and guar gum are the result of a difference only in the way their side chains are arranged.

Syneresis occurs when liquid weeps out of a gel over time, as happens in custards. Agar is prone to syneresis; water can be expelled merely by pressing on it. Some gels only experience syneresis after long periods of time. Many gels that are ruined by freezing (see freeze-thaw stability, below) tend to weep when thawed. Within a given hydrocolloid system, harder gels tend to weep more than softer ones. ${ }^{11,12}$

Gels that may be frozen and thawed repeatedly are called freeze-thaw stable. Many gels begin to degrade after freezing; only one freeze-thaw cycle is advised. When an unstable gel is frozen and later thawed, its texture and structural may be compromised by the physical changes. To offset this effect and promote freeze-thaw stability, a second thickening hydrocolloid may be added to the gel system.

The lower the viscosity reached at the respective stirring speed, the better fruit preparations with shear-thinning behaviour can be mixed with the galactomannans. To determine the viscosity, the shear rate is continuously increased and the resulting shear stress measured. Viscosity is the quotient of shear stress and shear rate $(\eta[\mathrm{Pas}]=\tau[\mathrm{Pa}]$ : $\mathrm{D}$ [s-1]). In the manufacture of fruit preparations with mixture of galactomannans, it is advantageous when a certain level of viscosity builds up in the idle state during the heating process, as a high heat viscosity counteracts the floating of the fruits by delaying floating. Floating can only be completely avoided if a yield point is already built up during the heating process. Substances with a yield point will not flow until external forces exceed their internal strength. When exposed to forces below the yield point the substances behave as though they were solids.

\section{Conclusions}

The filling temperature has an important influence on both the texture and the firmness of the final product. If a product, e.g. a fruit preparation, is produced and deposited, elastic gels of constantly high gel strengths are obtained as long as the filling temperature exceeds the setting temperature of the respective fruit preparation. If the filling temperature decreases, and, finally drops below the setting temperature, pre-gelation starts and part of the overall reachable gel strength is lost. At the same time, the lower the selected filling temperature, the more the viscosity of the gel texture of the pre-gelled fruit preparation increases. The enhancement in the values of the viscosity may be attributed to the arrangement of galactose: mannose series arrangement in the respective galactomannans and plays important role in the stabilization of the food formulations.

Many hydrocolloids gel when cooled. Sometimes these gels can be melted again, such as gelatin, and sometimes they cannot, such as the pectin in a jam. Methylcellulose forms a gel when heated that melts on cooling. Some thermally reversible gels show temperature hysteresis, that is, the setting temperature of the gel is lower than the temperature needed to melt the gel. This property can be very important to a chef. For example, agar sets around $35^{\circ} \mathrm{C}$ but melts at around $90^{\circ} \mathrm{C}$. The low set temperature makes agar easy to work with, and the high melt temperature allows agar preparations to be served hot. Thermally formed gels can also be slow set or snap set. Snap setting hydrocolloids, like gellan, gel instantly below their gelation temperature. As a rule of thumb, gelling hydrocolloids and thickening hydrocolloids can often be mixed to get the benefits of both (locust bean gum can be added to kappa carrageenan to give it a better texture, for example) without synergistic effects that will damage a recipe. Charged and uncharged hydrocolloids can also often be mixed without incident, like methylcellulose and alginate (for more information on synergistic effects. ${ }^{11,12}$

\section{ACKNOWLEDGEMENTS}

I am thankful to Mr. Peter Huber as Food technologist for his help in evaluating the fruit preparation and providing fruit preparation for the research from Austria.

\section{REFERENCES}

[1] Benhura M., 2002, Some properties of a polysaccharide preparation that is isolated from the fruit of Cordia abyssinica, Food Chemistry, 76(3):343-347

[2] Casas-Alencáster N. B., Pardo-García D. G., 2005, Texture profile analysis and stress relaxation properties of cross linked waxy maize starch-gellan mixed gels, Revista Mexicana de Ingeniería Química, 4 (1):107-121

[3] Cristina F., Noemi E. Z., 2000, Effect of freezing rate and frozen storage on starch - sucrose - hydrocolloid systems, Journal of the Science of Food and Agriculture, 2149-2158

[4] Eric D., 2003. Hydrocolloids at interfaces and the influence on the properties of dispersed systems, Food Hydrocolloids, 17(1): 25-39 
[5] Jaroslaw K., Leslaw J., Mariusz W., Bohdan A., 2004, Influence of selected hydrocolloids on triticale starch rheological properties, International journal of food science technology, 39(6):641-652

[6] Marcotte M., 2001, Rheological properties of selected hydrocolloids as a function of concentration and temperature, Food Research International, 34(8):695-703

[7] Ptaszek A., Berski W., Ptaszek P., Witczak T., Repelewicz U., Grzesik M,. 2009, Viscoelastic properties of waxy maize starch and selected non-starch hydrocolloids gels, Carbohydrate Polymers, 76(4): 567-577

[8] Sudhakar V., Singhal R. S., Kulkarni P. R., 1995, Effect of sucrose on starch-hydrocolloid interactions, Food Chemistry,
52(3): $281-284$

[9] Andreas H., 2008, Galactomannans and/or glucomannans for increasing the bioavailability of active substances, US patent $0206340 \mathrm{~A} 1$

[10] Anil Kumar A., Harjinder S., 2007. Recent advances in microencapsulation for industrial applications and targeted delivery. Trends in Food Science and Technology, 18: 240-251.

[11] Olivas G.I., Mattinson D.D., Barbosa- C'anovas, G.V., 2007. Alginate coatings for preservation of minimally processed 'Gala' apples. Postharvest Biology and Technology, 45: 89-96.

[12] Heinz A., 1991, Process of the preparation of fruit or spicy aerated foods, US patent 5000974 